Research Paper

\title{
Analytical solutions to the Stefan problem with internal heat generation
}

\author{
David McCord $^{a}$, John Crepeau ${ }^{\mathrm{a}, *}$, Ali Siahpush ${ }^{\mathrm{b}}$, João Angelo Ferres Brogin ${ }^{\mathrm{c}}$ \\ ${ }^{a}$ Department of Mechanical Engineering, University of Idaho, 875 Perimeter Drive, MS 0902, Moscow, ID 83844-0902, United States \\ ${ }^{\mathrm{b}}$ Department of Integrated Engineering, Southern Utah University, 351 W. University Blvd., Cedar City, UT 84720, United States \\ ${ }^{\mathrm{c}}$ Departamento de Engenharia Mecânica, São Paulo State University, Ilha Solteira, SP 15385-000, Brazil
}

\section{H I G H L I G H T S}

- A differential equation modeling the Stefan problem with heat generation is derived.

- The analytical solutions compare very well with the computational results.

- The system reaches steady-state faster for larger Stefan numbers.

- The interface location is proportional to the inverse square root of the heat generation.

\section{A R T I C L E I N F O}

\section{Article history:}

Received 15 February 2016

Accepted 24 March 2016

Available online 13 April 2016

\section{Keywords:}

Stefan problem

Internal heat generation

Solidification

Melting

\begin{abstract}
A B S T R A C T
A first-order, ordinary differential equation modeling the Stefan problem (solid-liquid phase change) with internal heat generation in a plane wall is derived and the solutions are compared to the results of a computational fluid dynamics analysis. The internal heat generation term makes the governing equations non-homogeneous so the principle of superposition is used to separate the transient from steadystate portions of the heat equation, which are then solved separately. There is excellent agreement between the solutions to the differential equation and the CFD results for the movement of both the solidification and melting fronts. The solid and liquid temperature profiles show a distinct difference in slope along the interface early in the phase change process. As time increases, the changes in slope decrease and the temperature profiles become parabolic. The system reaches steady-state faster for larger Stefan numbers and inversely, the time to steady-state increases as the Stefan number decreases.
\end{abstract}

(C) 2016 Published by Elsevier Ltd.

\section{Introduction}

Heat transfer driven by internal heat generation plays an important role in many engineering applications, including nuclear energy, systems involving joule or ohmic heating, geophysics and materials processing. Solid-liquid phase change, first described by Stefan [1] and known as the Stefan problem, is a nonlinear phenomenon, complicated by a time-dependent phase change interface. He solved the problem by assuming various temperature profiles and used the heat balance equation to determine the movement of the interface.

The Stefan problem is well-studied [2], and limited closed-form solutions exist. The text by Burmeister [3] provides an accessible similarity solution to the Stefan problem and shows that the phase change boundary increases as the square root of time. Yao and

\footnotetext{
* Corresponding author.

E-mail addresses: saltfirefan@gmail.com (D. McCord), crepeau@uidaho.edu (J. Crepeau), alisiahpush@suu.edu (A. Siahpush), jafb72@gmail.com (J.A. Ferres Brogin).
}

Prusa [4] review the heat transfer of freezing and melting, and Viskanta [5] discusses in detail solid-liquid phase change, specifically in metals. In many materials, a well-defined solidification front does not exist, but a so-called mushy zone, where the liquid and solid phases coexist. The mushy zone has been described by Worster [6].

The presence of internal heat generation further complicates the identification of a time-dependent solution. For materials which generate internal heat, very limited analytical or approximate solutions exist which relate the solidification or melting rate to the magnitude of the internal heat generation. Clearly the internal heat generation is coupled to the movement of the phase change front, accelerating melting and opposing solidification.

Chen et al. [7] proposed a simple transient conduction model with phase change in a material with internal heat generation using the lumped parameter technique and averaged properties. Their goal was to calculate the time when a nuclear fuel pin begins to melt as well as the time required to completely melt the fuel. Later, El-Genk and Cronenberg [8] used the successive approximation technique to study the effects of heat generation on the transient 


\section{Nomenclature}

$A_{n}, B_{n} \quad$ Fourier coefficients

$c_{p} \quad$ specific heat

$\Delta h_{f} \quad$ latent heat of fusion

$k$ thermal conductivity

$L$ distance from the centerline to the edge of the plane wall

$\dot{q} \quad$ volumetric internal heat generation

Q nondimensional internal heat generation

$s(t) \quad$ distance to the phase change front

St Stefan number (solid)

$t$ time

$T$ temperature

$T_{0} \quad$ constant surface temperature

$T_{m} \quad$ melt temperature

$x \quad$ distance

\section{Greek symbols}

$\alpha \quad$ thermal diffusivity

$\eta \quad$ nondimensional distance

$\phi \quad$ initial temperature profile

$\Phi \quad$ nondimensional initial temperature profile

$\lambda_{n}, \lambda_{n} \quad$ characteristic eigenvalues

$\theta \quad$ nondimensional temperature

$\rho \quad$ density

$\tau \quad$ nondimensional time

$\zeta(\tau) \quad$ nondimensional distance to phase change front

\begin{tabular}{ll}
\multicolumn{2}{l}{ Subscripts } \\
liq & liquid \\
sol & solid \\
ss & steady-state \\
tr & transient
\end{tabular}

freezing of molten nuclear fuel through a reactor shield plug. They found that their transient analysis was more accurate than previous steady-state analyses by more than a factor of two. For laminar flow of a fluid with internal heat generation in tubes, Kikuchi and Shigemasa [9] determined the heat transfer characteristics and calculated the Nusselt number and its behavior along the length of the tube. Numerical studies performed by Cheung et al. [10] detailed melting and freezing in a heat generating slab bounded by two semi-infinite cold walls. They stated that due to the highly nonlinear nature of the Stefan problem with internal heat generation, it was not feasible to seek even approximate analytical solutions. Chan et al. [11] described a phase change model where internal melting was induced by radiative transfer in semi-transparent materials. Chan and Hsu [12] used the enthalpy method to model the mushy zone in the phase change problem with uniform internal heat generation. They claimed that the mushy zone thickness increases as the solid melts.

Crepeau and Siahpush [13] found approximate solutions to the Stefan problem with internal heat generation using quasi-static methods, valid for Stefan numbers less than one. Crepeau et al. $[14,15]$ then compared those solutions to results from the computational analysis of Spotten [16] and showed good agreement for Stefan numbers less than one. Yu et al. [17] used perturbation methods to solve the Stefan problem with internal heat generation, and showed good results for Stefan numbers less than one and reasonable results Stefan numbers one and higher. An and Su [18] used a lumped parameter model obtained through two-point Hermite approximations for integrals. They found excellent agreement with computational models using the enthalpy method. An et al. [19] used a finite difference technique to model the melting process, driven by internal heat generation, in a nuclear fuel rod. Shrivistava et al. [20] performed experimental studies of melting with internal heat generation and compared their results with computational modeling and showed favorable correlation.

\section{Problem description}

The solid-liquid phase change problem with internal heat generation in a symmetric, plane-wall geometry is considered. A schematic of the geometry, cut along the centerline is presented in Fig. 1. The phase change is driven by internal heat generation, $\dot{q}$, in both the solid and liquid phases. The temperature at which the phase change occurs, i.e., the fusion temperature, is denoted by $T_{m}$, and the wall is held at a constant temperature, $T_{0}$, which is below the fusion temperature. The temperature gradient, by symmetry, at the centerline is equal to zero.
For the solution to the problem, the following assumptions were made: (1) the internal heat generation is constant and the same in both the solid and liquid phases; (2) the material properties in both phases are constant, uniform and equal; (3) heat is transferred solely by conduction and there is no convection in the liquid phase; and (4) the phase change occurs at a single, constant temperature, $T_{m}$, so there is no mushy zone at the interface between the phases.

The governing equation in both the solid and liquid phases is given by the heat conduction equation [21],

$\frac{\partial^{2} T(x, t)}{\partial x^{2}}+\frac{\dot{q}}{k}=\frac{1}{\alpha} \frac{\partial T(x, t)}{\partial t} \quad 0 \leqslant x \leqslant \infty, \quad 0 \leqslant t \leqslant \infty$

with the boundary and initial conditions in the liquid phase given by,

$$
\begin{aligned}
& \frac{\partial T_{\text {liq }}(0, t)}{\partial x}=0 \quad T_{\text {liq }}(s(t), t)=T_{m} \\
& T_{\text {liq }}(x, 0)=\phi_{\text {liq }}(x)
\end{aligned}
$$

and in the solid phase the boundary and initial conditions are,

$$
\begin{aligned}
& T_{\text {sol }}(s(t), t)=T_{m} \quad T_{\text {sol }}(L, t)=T_{0} \\
& T_{\text {sol }}(x, 0)=\phi_{\text {sol }}(x)
\end{aligned}
$$

There is conduction heat transfer along the interface between the solid and liquid phases, as well as latent heat. The governing equation along the interface is given as [22],

$\left.k_{\text {liq }} \frac{\partial T_{\text {liq }}(x, t)}{\partial x}\right|_{x=s}+\rho_{\text {sol }} \Delta h_{f} \frac{d s(t)}{d t}=\left.k_{\text {sol }} \frac{\partial T_{\text {sol }}(x, t)}{\partial x}\right|_{x=s(t)}$

The governing equations are then nondimensionalized in order to simplify the solution process. The following terms are introduced,

$$
\begin{aligned}
& \eta=\frac{x}{L}, \quad \tau=\frac{\alpha t}{L^{2}}, \quad \theta(\eta, \tau)=\frac{T(x, t)-T_{0}}{T_{m}-T_{0}}, \quad \zeta(\tau)=\frac{s(t)}{L}, \\
& S t=\frac{c_{p}\left(T_{m}-T_{0}\right)}{\Delta h_{f}}, \quad Q=\frac{\dot{q} L^{2}}{k\left(T_{m}-T_{0}\right)}
\end{aligned}
$$

Here, $\eta$ is the nondimensional distance, $\tau$ is the Fourier number (nondimensional time), $\theta(\eta, \tau)$ is the nondimensional temperature, $\zeta(\tau)$ is the nondimensional distance from the centerline to the phase change front, $S t$ is the Stefan number of the solid, and $Q$ is the nondimensional internal heat generation. By substituting these variables into the governing equations, the heat equation becomes, 


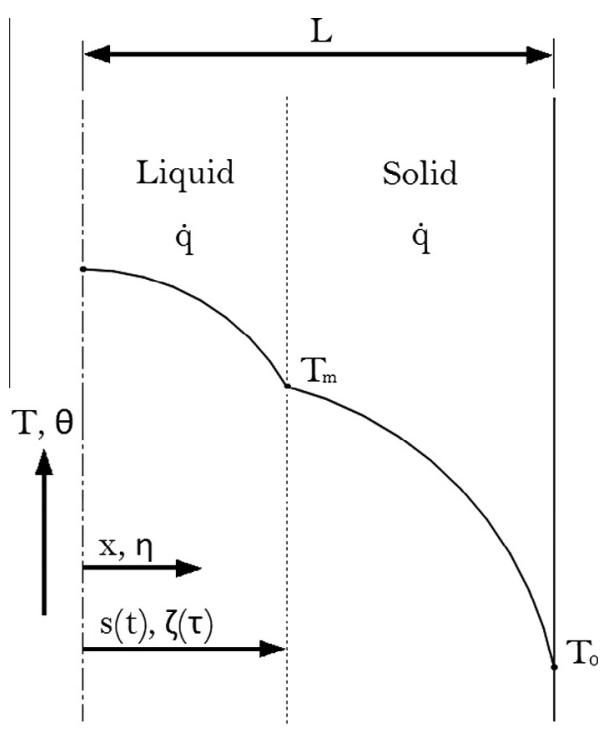

Centerline

Fig. 1. Schematic of the solid-liquid phase change problem with internal heat generation in a symmetric, plane wall. The slope of the temperature at the centerline is zero.

$\frac{\partial^{2} \theta(\eta, \tau)}{\partial \eta^{2}}+Q=\frac{\partial \theta(\eta, \tau)}{\partial \tau}$

where the boundary and initial conditions in the liquid phase are,

$\frac{\partial \theta_{\text {liq }}(0, \tau)}{\partial \eta}=0, \quad \theta_{\text {liq }}(\zeta(\tau), \tau)=1, \quad \theta_{\text {liq }}(\eta, 0)=\Phi_{\text {liq }}(\eta)$

and in the solid phase the boundary and initial conditions are,

$\theta_{\text {sol }}(\zeta(\tau), \tau)=1, \quad \theta_{\text {sol }}(1, \tau)=0, \quad \theta_{\text {sol }}(\eta, 0)=\Phi_{\text {sol }}(\eta)$

The nondimensional interface equation is,

$\left.\frac{\partial \theta_{\text {liq }}(\eta, \tau)}{\partial \eta}\right|_{\eta=\zeta}+\frac{1}{S t} \frac{d \zeta(\tau)}{d \tau}=\left.\frac{\partial \theta_{\text {sol }}(\eta, \tau)}{\partial \eta}\right|_{\eta=\zeta}$
In order to solve Eq. (9) the temperature profiles in the solid and liquid phases must be known. Since Eq. (1) is a nonhomogeneous partial differential equation the method of superposition is used to obtain steady-state and transient equations, which are then solved by separation of variables. The original equation is split into a homogeneous partial differential equation in $\eta$ and $\tau$, and a steady-state ordinary differential equation which are solved separately, then superposed to obtain a final solution to the original differential equation [23].

First, the liquid phase is considered. The temperature profile is separated into transient and steady-state profiles,

$\theta_{\text {liq }}(\eta, \tau)=\theta_{\text {liq }, t r}(\eta, \tau)+\theta_{\text {liq,ss }}(\eta), \quad 0 \leqslant \eta \leqslant \zeta(\tau)$

Substituting Eq. (10) into Eq. (6) results in,

$\frac{\partial^{2} \theta_{\text {liq,tr }}(\eta, \tau)}{\partial \eta^{2}}+\frac{d^{2} \theta_{\text {liq,ss }}(\eta)}{d \eta^{2}}+Q=\frac{\partial \theta_{\text {liq,tr }}(\eta, \tau)}{\partial \tau}$

For the associated boundary and initial conditions, Eq. (10) is substituted into Eq. (7). The result is,

$\frac{\partial \theta_{\text {liq }, t r}(0, \tau)}{\partial \eta}+\frac{d \theta_{\text {liq,ss }}(0)}{d \eta}=0$
$\theta_{\text {liq,tr }}(\zeta(\tau), \tau)+\theta_{\text {liq }, s s}(\zeta(\tau))=1$
$\theta_{\text {liq, }, t r}(\eta, 0)+\theta_{\text {liq }, s s}(\eta)=\Phi_{\text {liq }}(\eta)$

The transient and steady-state problems summed together must satisfy Eqs. (10)-(12). The governing equation of the transient portion of the problem is homogeneous and is given as,

$\frac{\partial^{2} \theta_{\text {liq,tr }}(\eta, \tau)}{\partial \eta^{2}}=\frac{\partial \theta_{\text {liq,tr }}(\eta, \tau)}{\partial \tau}$

with boundary and initial conditions,

$\frac{\partial \theta_{\text {liq,tr }}(0, \tau)}{\partial \eta}=0, \quad \theta_{\text {liq,tr }}(\zeta(\tau), \tau)=0$,

$\theta_{\text {liq }, t r}(\eta, 0)=\Phi_{\text {liq }}(\eta)-\theta_{\text {liq }, s s}(\eta)$

Subtracting Eq. (13) from Eq. (11) leaves the governing equation for the steady-state problem,

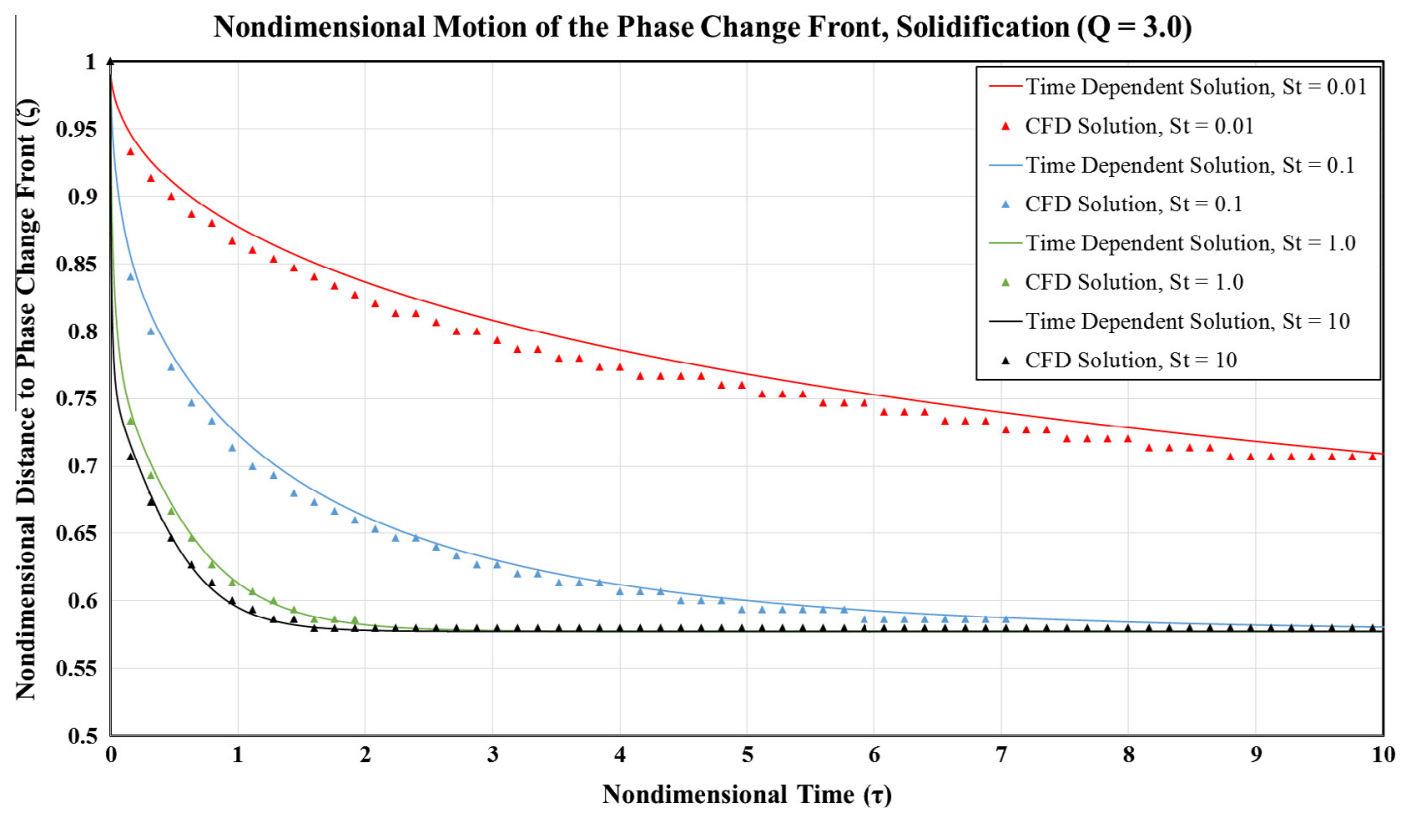

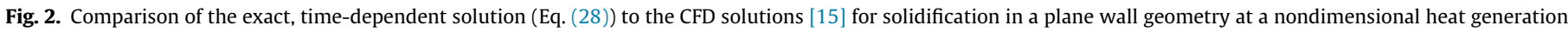
of $Q=3.0$. 

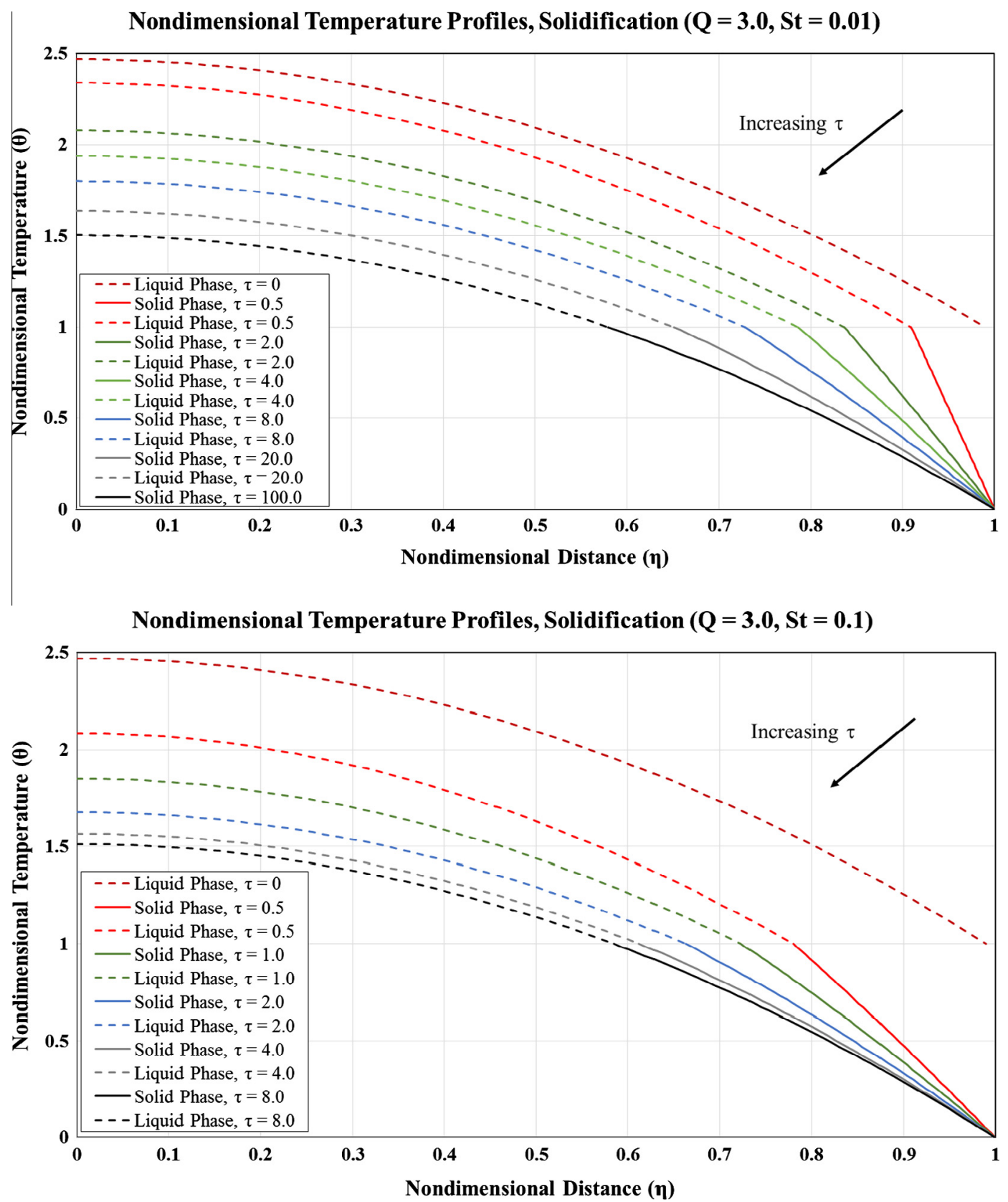

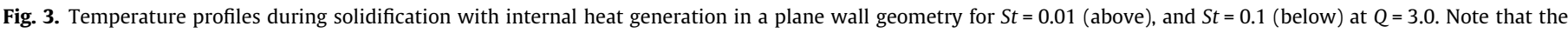
differences in slopes of the temperature profiles at the interface $(\theta=1)$ between the solid and liquid phases diminish with increasing $\tau$.

$\frac{d^{2} \theta_{\text {liq,ss }}(\eta)}{d \eta^{2}}=-Q$

with the boundary conditions for the steady-state portion given by,

$\frac{d \theta_{\text {liq,ss }}(0)}{d \eta}=0, \quad \theta_{\text {liq,ss }}(\zeta(\tau))=1$

The sum of the transient and steady-state portions of the problem yields the original set of equations for the temperature profile in the liquid phase, Eqs. (11) and (12).

Integrating Eq. (15) twice and applying the boundary conditions produces the temperature distribution,

$\theta_{\text {liq }, s s}(\eta)=\frac{1}{2} Q\left(\zeta^{2}(\tau)-\eta^{2}\right)+1$
The transient portion of the temperature distribution in the liquid phase, Eqs. (13) and (14) can be solved using standard separation of variables techniques [24]. The resulting temperature profile is given as,

$$
\begin{aligned}
& \theta_{\text {liq }}(\eta, \tau)=\theta_{\text {liq }, t r}(\eta, \tau)+\theta_{\text {liq, }, s s}(\eta), \quad 0 \leqslant \eta \leqslant \zeta(\tau) \\
& \theta_{\text {liq }}(\eta, \tau)=\sum_{n=1}^{\infty} A_{n} \cos \left(\lambda_{n} \eta\right) e^{-\lambda_{n}^{2} \tau}+\frac{1}{2} Q\left(\zeta^{2}(\tau)-\eta^{2}\right)+1 \\
& A_{n}=\frac{2}{\zeta(\tau)} \int_{0}^{\zeta}\left[\Phi_{\text {liq }}(\eta)-\theta_{\text {liq }, s s}(\eta)\right] \cos \left(\lambda_{n} \eta\right) d \eta, \\
& \lambda_{n}=\frac{(2 n-1) \pi}{2 \zeta(\tau)}, \quad n=1,2, \ldots
\end{aligned}
$$

Next, a similar process is used for the solid phase, but with appropriate boundary and initial conditions. The temperature is given by transient and steady-state components, 
$\theta_{\text {sol }}(\eta, \tau)=\theta_{\text {sol.tr }}(\eta, \tau)+\theta_{\text {sol.ss }}(\eta), \quad \zeta(\tau) \leqslant \eta \leqslant 1$

As before, the transient portion of the equation is given by,

$\frac{\partial^{2} \theta_{\text {sol }, t r}(\eta, \tau)}{\partial \eta^{2}}=\frac{\partial \theta_{\text {sol,tr }}(\eta, \tau)}{\partial \tau}$

with the boundary and initial conditions,

$\theta_{\text {sol }, \text { tr }}(\zeta(\tau), \tau)=0, \quad \theta_{\text {sol,tr }}(1, \tau)=0$

$\theta_{\text {sol,tr }}(\eta, 0)=\Phi_{\text {sol }}(\eta)-\theta_{\text {sol,ss }}(\eta)$

The steady-state component of the nondimensional temperature is,

$\frac{d^{2} \theta_{\text {sol }, s s}(\eta)}{d \eta^{2}}=-Q$

with boundary conditions,

$\theta_{\text {sol }, s s}(\zeta(\tau))=1, \quad \theta_{\text {sol,ss }}(1)=0$

Integrating Eq. (22) twice and applying the boundary conditions in Eq. (23) results in,

$$
\theta_{\text {sol }, s s}(\eta)=-\frac{1}{2} Q \eta^{2}+\frac{1+\frac{1}{2} Q\left(\zeta^{2}(\tau)-1\right)}{\zeta(\tau)-1}(\eta-1)+\frac{1}{2} Q
$$

For the transient component, Eq. (20) is solved using separation of variables similar to the liquid phase. The complete form of the temperature profile in the solid region is then,

$$
\begin{aligned}
& \theta_{\text {sol }}(\eta, \tau)=\theta_{\text {sol, }, \text { r }}(\eta, \tau)+\theta_{\text {sol }, \text { s }}(\eta), \quad \zeta(\tau) \leqslant \eta \leqslant 1 \\
& \theta_{\text {sol }}(\eta, \tau)=\sum_{n=1}^{\infty} B_{n}\left[-\tan \left(\lambda_{n}\right) \cos \left(\lambda_{n} \eta\right)+\sin \left(\lambda_{n} \eta\right)\right] e^{-\lambda_{n}^{2} \tau} \\
& \quad-\frac{1}{2} Q \eta^{2}+\frac{1+\frac{1}{2} Q\left(\zeta(\tau)^{2}-1\right)}{\zeta(\tau)-1}(\eta-1)+\frac{1}{2} Q \\
& B_{n}=\frac{2 \cos ^{2}\left(\lambda_{n}\right)}{1-\zeta(\tau)} \int_{\zeta}^{1}\left[\Phi_{\text {sol }}(\eta)-\theta_{\text {sol }, s s}(\eta)\right]\left[-\tan \left(\lambda_{n}\right) \cos \left(\lambda_{n} \eta\right)\right. \\
& \left.\quad+\sin \left(\lambda_{n} \eta\right)\right] d \eta \\
& \lambda_{n}=\frac{n \pi}{1-\zeta(\tau)}, \quad n=1,2, \ldots
\end{aligned}
$$
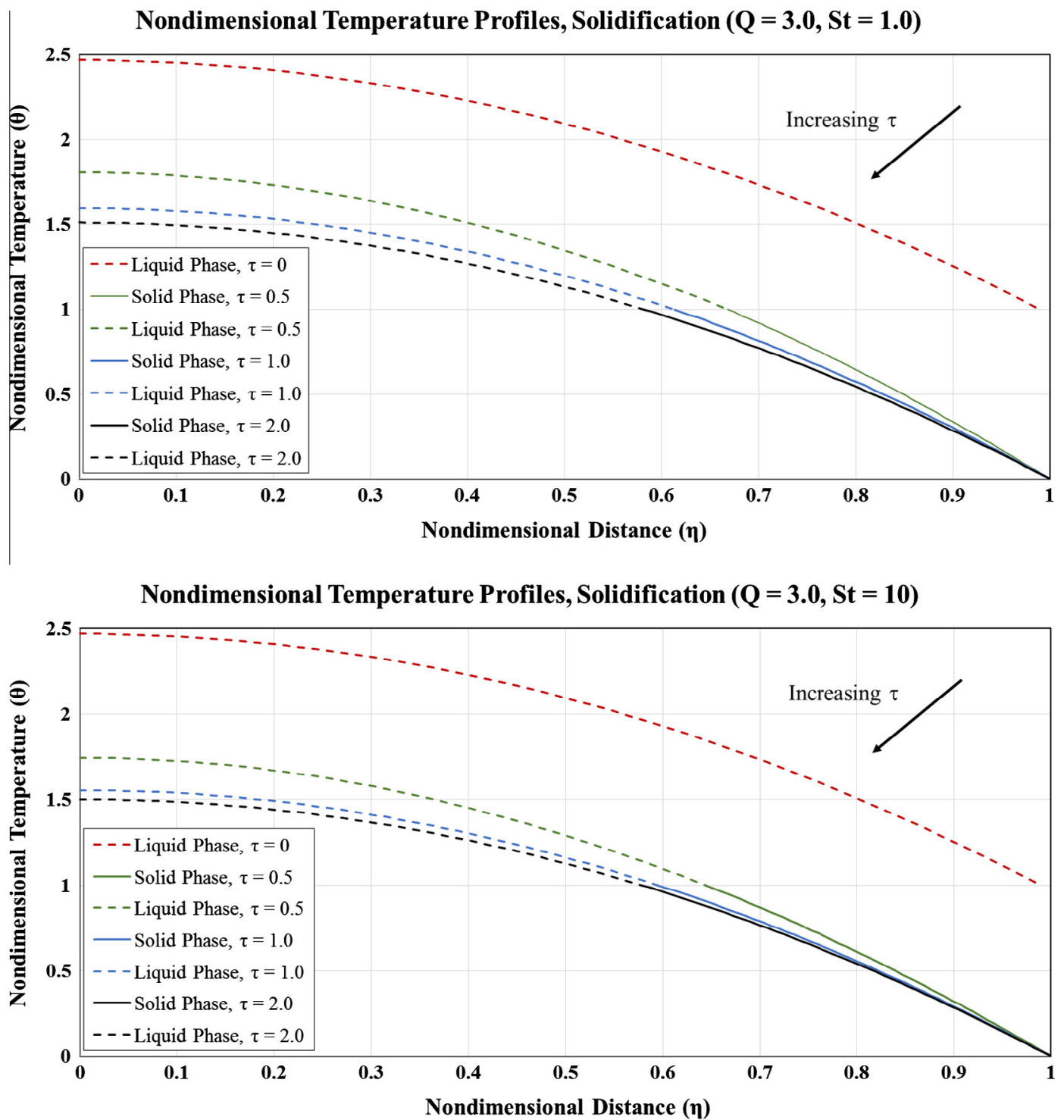

Fig. 4. Temperature profiles during solidification with internal heat generation in a plane wall geometry for $S t=1.0$ (above), and $S t=10.0$ (below) at $Q=3.0$ 
With the time-dependent temperature profiles in the solid and liquid phases determined, the partial derivatives with respect to the nondimensional distance can be calculated and used in the interface equation, Eq. (9).

For the liquid region, Eq. (18), the temperature gradient is,

$\left.\frac{\partial \theta_{\text {liq }}(\eta, \tau)}{\partial \eta}\right|_{\eta=\zeta(\tau)}=\sum_{n=1}^{\infty}(-1)^{n} A_{n} \lambda_{n} e^{-\lambda_{n}^{2} \tau}-Q \zeta(\tau)$

where $A_{n}$ and $\lambda_{n}$ are given in Eq. (18).

For the solid region, Eq. (25), the temperature gradient is calculated as,

$$
\begin{aligned}
\left.\frac{\partial \theta_{\text {sol }}(\eta, \tau)}{\partial \eta}\right|_{\eta=\zeta(\tau)}= & \sum_{n=1}^{\infty} B_{n} \lambda_{n}\left[\tan \left(\lambda_{n}\right) \sin \left(\lambda_{n} \zeta(\tau)\right)\right. \\
& \left.+\cos \left(\lambda_{n} \zeta(\tau)\right)\right] e^{-\lambda_{n}^{2} \tau}-Q \zeta(\tau) \\
& +\frac{1+\frac{1}{2} Q\left(\zeta^{2}(\tau)-1\right)}{\zeta(\tau)-1}
\end{aligned}
$$

where $B_{n}$ and $\lambda_{n}$ are given in Eq. (25).

Substituting Eqs. (26) and (27) into the interface equation, Eq. (9) yields,

$$
\begin{aligned}
\sum_{n=1}^{\infty}(-1)^{n} A_{n} \lambda_{n} e^{-\lambda_{n}^{2} \tau}+\frac{1}{S t} \frac{d \zeta(\tau)}{d \tau}= & \sum_{n=1}^{\infty} B_{n} \lambda_{n}\left[\tan \left(\lambda_{n}\right) \sin \left(\lambda_{n} \zeta(\tau)\right)\right. \\
& \left.+\cos \left(\lambda_{n} \zeta(\tau)\right)\right] e^{-\lambda_{n}^{2} \tau} \\
& +\frac{1+\frac{1}{2} Q\left(\zeta^{2}(\tau)-1\right)}{\zeta(\tau)-1}
\end{aligned}
$$

The initial conditions are dependent on whether the system is solidifying or melting. Eq. (28) governs the movement of the solid-liquid phase change front and its dependence on two parameters, the Stefan number, $S t$, and the nondimensional heat generation, $Q$. It is an unusual equation in the sense that two of the terms are infinite series which exponentially decay in time. For fixed values of $S t$ and $Q$ Eq. (28) can be integrated numerically, giving the behavior of the solid-liquid phase change front in time.

\section{Results}

Solutions to Eq. (28) are presented for both solidification and melting. The forward Euler integration method [25] was used to solve Eq. (28) with the appropriate boundary conditions. These solutions are then compared to computational fluid dynamics solutions $[14,16]$. From CFD, the energy equations were solved using ANSYS Fluent [26] for the phase change based on the enthalpyporosity method described in Voller and Prakash [27]. The method used an implicit, standard pressure-based solver with an unsteady, first-order upwind technique which is typically adequate provided small time steps are used and smooth changes exist in the solution behavior. An iterative time advancement solver option was used since it provided a more stable solution convergence. A fixed grid was established initially, then refined. Further grid refinement did not alter the results. For additional details, see Spotten [16].

\subsection{Steady-state}

The steady-state solution of Eq. (28) is readily obtainable and is given by,

$$
\zeta(\tau \rightarrow \infty)=\sqrt{1-\frac{2}{Q}}
$$

This matches the steady-state value calculated previously using approximate methods [13]. It also gives the minimum value of $Q$ $(>2)$ for which melting occurs in this geometry.

\subsection{Solidification}

For the solidification process, at the outset, the material is completely in the liquid phase, which ranges from $0 \leqslant \eta \leqslant \zeta(\tau)$ and has a temperature distribution given by Eq. (17). The initial condition is $\zeta(\tau=0)=1$.

Fig. 2 presents the numerical solution of Eq.(28) for $Q=3.0$ with the CFD solutions for values of the Stefan number ranging from $S t=0.01$ to $S t=10.0$ in decadal increments. The Stefan number is the ratio of the sensible heat to the latent heat, and for values less than one the latent heat dominates and the phase change process

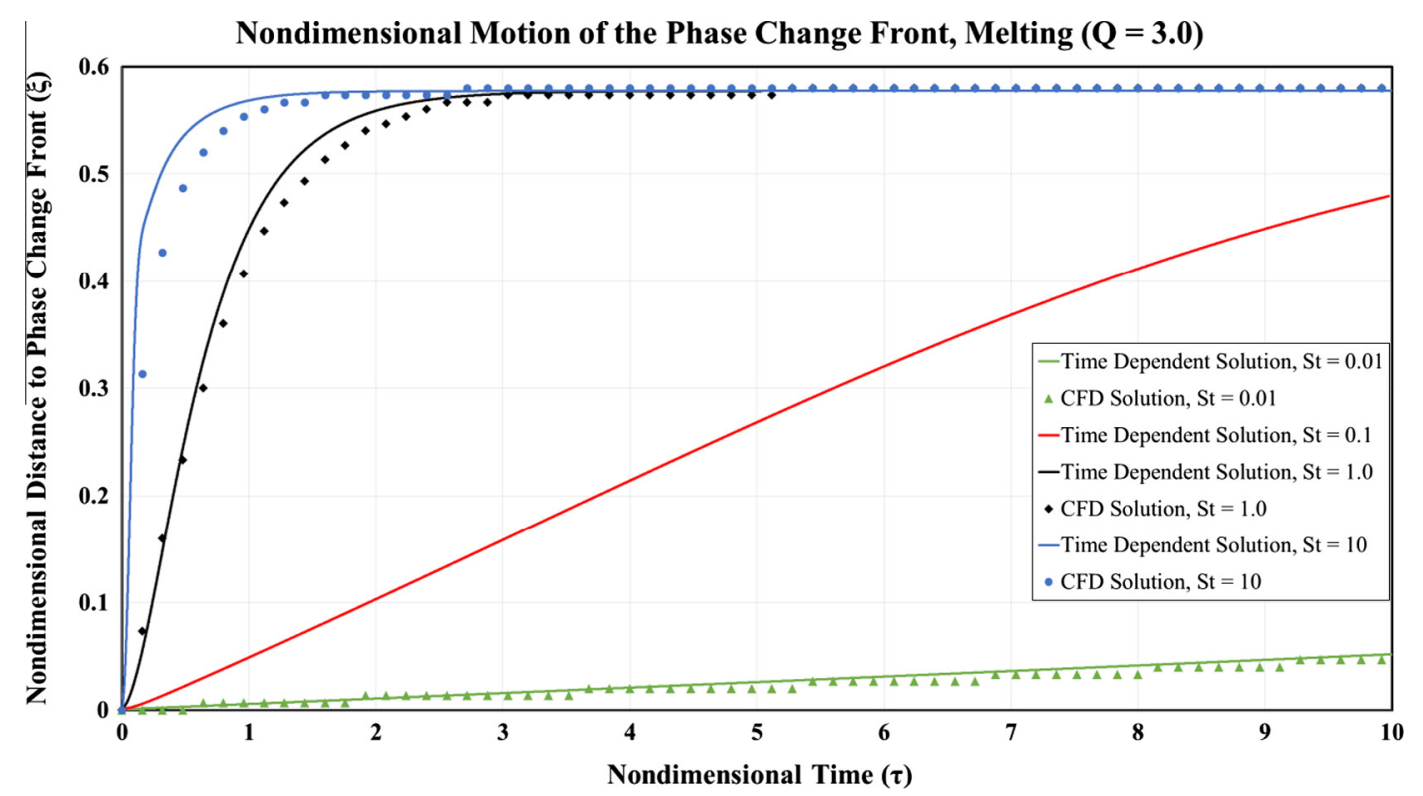

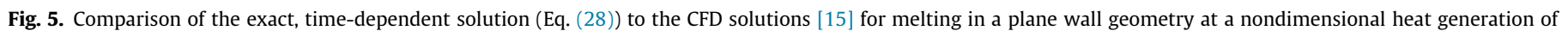
$Q=3.0$. 

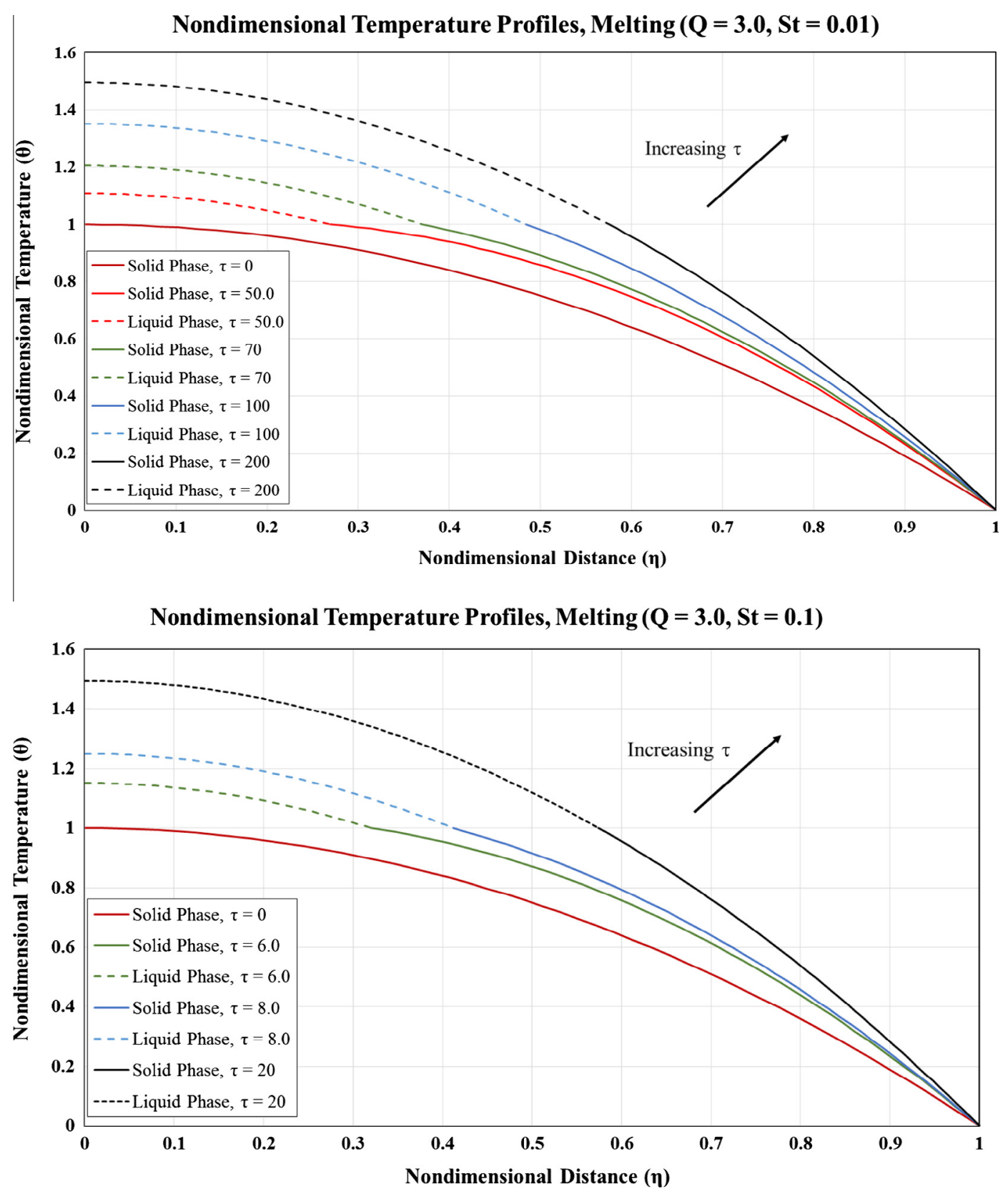

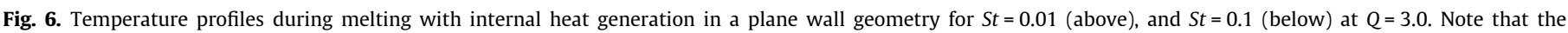
difference in slopes of the temperature profiles at the interface $(\theta=1)$ between the solid and liquid phases diminish with increasing $\tau$.

is relatively slow. In Fig. 2 at $S t=0.01$, this is the case and the system does not reach steady-state until about $\tau=100$. As the Stefan number increases, the relative effect of the latent heat decreases and the interface moves faster towards steady-state which can be seen in the $S t=1.0$ and $S t=10.0$ curves. The comparison between the solutions of Eq. (28) and the CFD results is excellent, especially at higher $S t$ where the approximate results of Crepeau et al. [14] showed poor agreement. According to Eq. (29), for $Q=3.0$, the steady-state location of the interface is $\zeta(\tau \rightarrow \infty) \approx$ 0.577 , and this matches the results shown in the figure.

Fig. 3 shows how the temperature profiles in the solid and liquid phases vary in time for $S t=0.01$ and $S t=0.1$ and $Q=3.0$. The dashed lines correspond to the liquid phase and the solid lines to the solid phase. For the $S t=0.01$ curves, the front moves slowly, and there is a distinct difference in the slopes of the temperature profiles of the two phases at the interface $(\theta=1)$ for $\tau \leqslant 8.0$. This pronounced, lingering difference in slopes occurs because the sensible heat is small compared to the latent heat, and the material does not respond sufficiently fast to the change in phase. As time increases the slopes of the two phases at the interface approach each other until the system reaches steady-state. At this point the temperature profile is completely parabolic. For $S t=0.1$, the difference in slopes at the interface can be seen for $\tau \leqslant 2.0$, and as time increases the system reaches steady-state. Fig. 4 shows the temperature profiles for $S t=1.0$ and $S t=10.0$. There is only a very small difference in the slope of the temperature profiles at the interface for $\tau=0.5$ and the system reaches steady-state relatively quickly, at around $\tau=2.0$.

\subsection{Melting}

For melting, the material begins in the solid phase and then starts to melt along the centerline. The initial temperature distribution is given by Eq. (24), and the initial condition is $\zeta(\tau=0)=0$. 

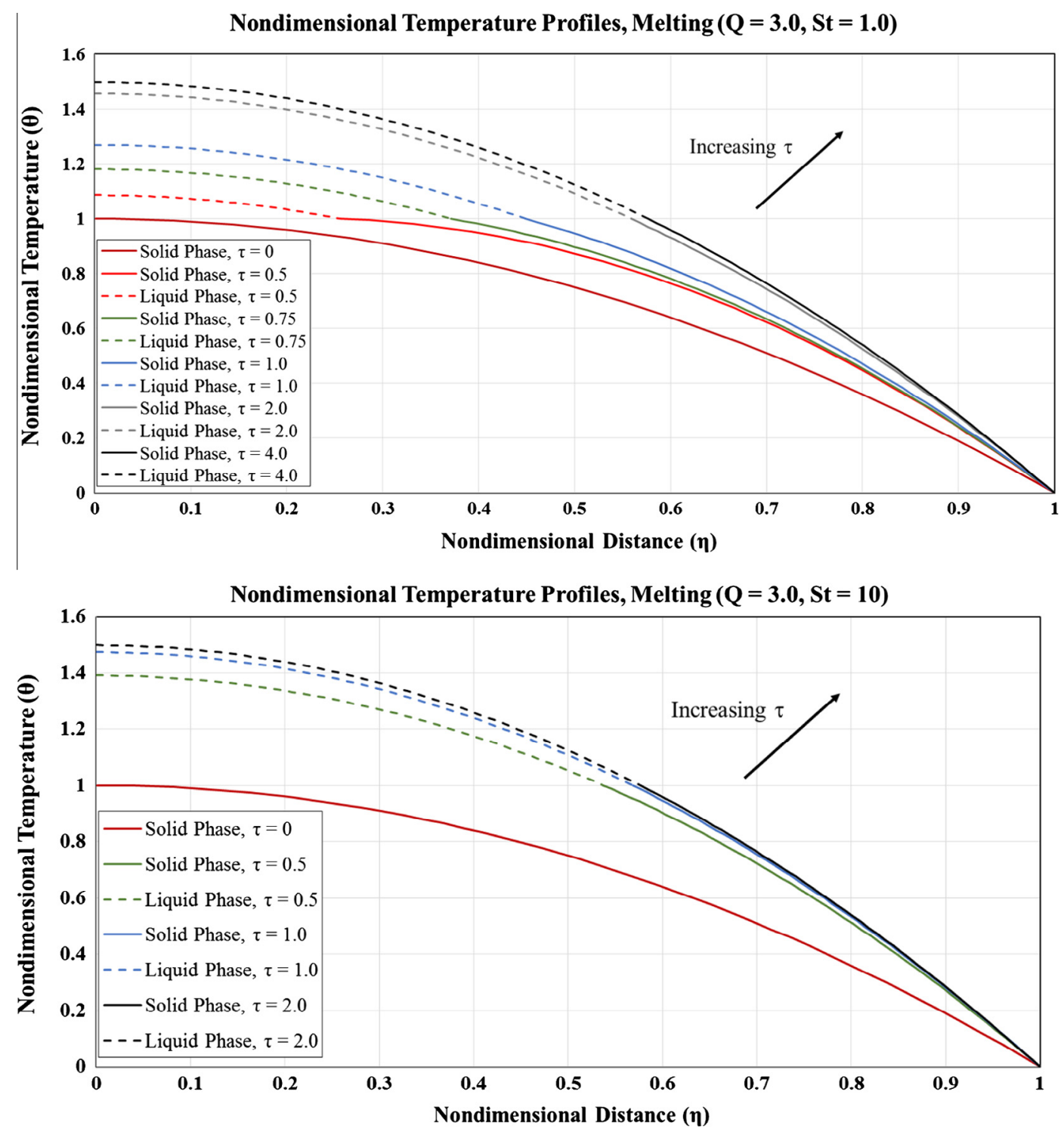

Fig. 7. Temperature profiles during melting with internal heat generation in a plane wall geometry for $S t=1.0$ (above), and $S t=10.0$ (below) at $Q=3.0$.

Fig. 5 gives the movement of the phase change front, also at $Q=3.0$ and compares the exact solution of Eq. (28) with the CFD solutions. At $S t=0.01$, again the phase change front moves slowly, and the agreement between the solution of Eq. (28) and the CFD results is very good. The motion of the front for $S t=0.01$ and $S t=0.1$ also compare favorably with the approximate solutions given in [14]. For $S t=1.0$ and $S t=10.0$ there is good agreement between Eq. (28) and the computational solutions. At these higher Stefan numbers, the exact solutions are a marked improvement over the approximate solutions.

The temperature profiles of the solid and liquid phases for the melting process at $S t=0.01$ and $S t=0.1$ are shown in Fig. 6. As before, the phase change front moves slowly, and there are distinct changes in the slopes of the temperature profiles at the interface $(\theta=1)$ for $\tau \leqslant 70$ at $S t=0.01$, and $\tau \leqslant 8.0$ at $S t=0.1$. As $\tau$ increases the temperature profiles reach their steady-state, parabolic profiles. In Fig. 7, the temperature profiles for $S t=1.0$ and $S t=10.0$ are presented. For $S t=1.0$, the temperature profiles become parabolic at around $\tau=1.0$ and for $S t=10$ there is very little change in the slopes of the temperature profiles for $\tau \geqslant 0.5$.

\section{Conclusions}

An exact solution to the problem of solid-liquid phase change driven by internal heat generation is derived. The results compare very favorably with CFD solutions for both solidification and melting. The key step to obtaining the exact solution to the nonhomogeneous problem was to use the method of superposition to separate the problem into transient and steady-state parts, solve each portion separately, then combining the solutions for the final result. The temperature profiles at low Stefan numbers show a distinct change in the slope at the interface between the solid and liquid phases for low values of the nondimensional time, $\tau$. As $\tau$ increases and the system reaches steady-state, the temperature profiles become parabolic.

\section{References}

[1] J. Stefan, Über die Theorie der Eisbildung, insbesondere über die Eisbildung im Polarmeere, Sitzungsberichte der k.k. Akademie der Wissenschaften in Wien, Mathematische-Naturwissenschaften, Abteilung II, 1889, pp. 965-983.

[2] L.I. Rubenstein, The Stefan Problem, AMS Pubs., Providence, 1971. 
[3] L.C. Burmeister, Convective Heat Transfer, Wiley, New York, 1993, pp. 81-88.

[4] L.S. Yao, J. Prusa, Melting and freezing, Adv. Heat Transfer 19 (1989) 1-95.

[5] R. Viskanta, Heat transfer during melting and solidification of metals, J. Heat Transfer 110 (1988) 1205-1219.

[6] M.G. Worster, Natural convection in a mushy layer, J. Fluid Mech. 224 (1991) 335-359.

[7] W.L. Chen, M. Ishii, M.A. Grolmes, Simple heat conduction model with phase change for reactor fuel pin, Nucl. Sci. Eng. 60 (1976) 452-460.

[8] M. El-Genk, A.W. Cronenberg, An assessment of fuel freezing and drainage phenomena in a reactor shield plug following a core disruptive accident, Nucl. Eng. Des. 47 (1978) 195-225.

[9] Y. Kikuchi, Y. Shigemasa, Liquid solidification in laminar tube flow with internal heat generation, Nucl. Eng. Des. 75 (1982) 73-80.

[10] F.B. Cheung, T.C. Chawla, D.R. Pedersen, The effects of heat generation and wal interaction on freezing and melting in a finite slab, Int. J. Heat Mass Transfer 27 (1984) 29-37.

[11] S.H. Chan, D.H. Cho, G. Kocamustafaogullari, Melting and solidification with internal radiative transfer - a generalized phase change model, Int. J. Heat Mass Transfer 26 (1983) 621-633.

[12] S.H. Chan, K.Y. Hsu, Applications of a generalized phase change model for melting and solidification of materials with internal heat generation, in: Proceedings of the AIAA 19th Thermophysics Conference, Snowmass, CO, 1984.

[13] J. Crepeau, A. Siahpush, Approximate solutions to the Stefan problem with internal heat generation, Heat Mass Transfer 44 (2008) 787-794.

[14] J. Crepeau, A. Siahpush, B. Spotten, On the Stefan problem with volumetric energy generation, Heat Mass Transfer 46 (2009) 119-128.

[15] J. Crepeau, A. Siahpush, Solid-liquid phase change driven by internal heat generation, C. R. Mécaniq. 340 (2012) 471-476.
[16] B. Spotten, Computational Fluid Dynamic Simulations of the Stefan Problem with Internal Heat Generation, MS Thesis, University of Idaho, Moscow, ID, 2008.

[17] Z.-T. Yu, L.-W. Fan, Y.-C. Hu, K.-F. Cen, Perturbation solutions to heat conduction in melting or solidification with heat generation, Heat Mass Transfer 46 (2010) 479-483.

[18] C. An, J. Su, Lumped parameter model for one-dimensional melting in a slab with volumetric heat generation, Appl. Therm. Eng. 60 (2013) (2013) 387-396.

[19] C. An, F.C. Moreira, J. Su, Thermal analysis of the melting process in a nuclear fuel rod, Appl. Therm. Eng. 68 (2014) 133-143.

[20] A. Shrivistava, B. Williams, A.S. Siahpush, B. Savage, J. Crepeau, Numerical and experimental investigation of melting with internal heat generation within cylindrical enclosures, Appl. Therm. Eng. 67 (2014) 587-596.

[21] F.P. Incropera, D.P. DeWitt, Introduction to Heat Transfer, fourth ed., Wiley, New York, 2002, pp. 100-110.

[22] D. Poulikakos, Conduction Heat Transfer, Prentice-Hall, Englewood Cliffs, 1994 p. 224.

[23] G.E. Myers, Analytical Methods in Conduction Heat Transfer, second ed., AMCHT Publications, Madison, 1998, pp. 141-142.

[24] E. Kreyszig, Advanced Engineering Mathematics, ninth ed., Wiley, New York, 2005, pp. 540-546.

[25] Matlab, release 2012b, The MathWorks, Inc., Natick, MA, USA

[26] ANSYS Fluent, release 6.3.28, User Manual, Ansys, Inc., Canonsburg, PA, USA, 2008.

[27] R. Voller, C. Prakash, A fixed-grid numerical modeling methodology for convection-diffusion mushy region phase-change problems, Int. J. Heat Mass Transfer 30 (1987) 1709-1720. 